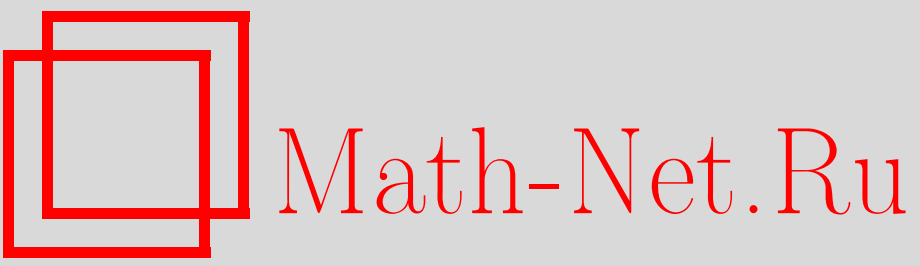

Е. Л. Кузнецова, Моделирование теплового состояния композиционных материалов на основе универсального закона разложения связующих, Вестн. Сам. гос. техн. ун-та. Сер. Физ.-мат. науки, 2010, выпуск 5(), 170-178

DOI: https://doi.org/10.14498/vsgtu746

Использование Общероссийского математического портала Math-Net.Ru подразумевает, что вы прочитали и согласны с пользовательским соглашением

http: //www. mathnet.ru/rus/agreement

Параметры загрузки:

IP : 54.166 .219 .16

26 апреля 2023 г., 18:13:01 
УДК 517.958+536.2(075)

\section{МОДЕЛИРОВАНИЕ ТЕПЛОВОГО СОСТОЯНИЯ КОМПОЗИЦИОННЫХ МАТЕРИАЛОВ НА ОСНОВЕ УНИВЕРСАЛЬНОГО ЗАКОНА РАЗЛОЖЕНИЯ СВЯЗУЮЩИХ}

\section{Е. Л. Кузнецова}

Московский авиационный институт (государственный технический университет), 125993, Москва, А-80, ГСП-3, Волоколамское шоссе, 4.

E-mail: lareyna@mail.ru

На основе идентификащии закона разложения связующих композиционных материалов предложена математическая модель и метод численного решения задач о тепловом состоянии больиинства композиционных материалов в условиях существенно нестационарного высокотемпературного нагружения. Полученный закон разложения связующих позволил в задачах о тепловом состоянии композиционных материалов не рассматривать химическую кинетику в области разложения связующего. Анализ численных резулътатов подтверждает предложенный подход $к$ моделированию теплового состояния композичионных материалов.

Ключевые слова: композиционные материаль, тонковолокнистые наполнители, связующие смолы, тепломассоперенос, математическое моделирование, численнъье методы.

Введение. Композиционные материалы широко применяются в качестве теплозащитных материалов благодаря своим уникальным свойствам, вытекающим из технологии их изготовления. Матрица из тонковолокнистого наполнителя (стекло-, абсо-, угле- и т. д. волокон) пропитывается связующими смолами, легко разлагающимися при умеренных температурах. При использовании таких материалов в качестве теплозащитных при гиперзвуковых скоростях полета (числа Маха $M \geqslant 5 \div 6$ ) летательных аппаратов тепловые потоки от высокотемпературных пограничных слоев поглощаются за счёт следующих факторов: объёмной теплоёмкости до $500 \mathrm{~K}$; эндотермических реакций разложения связующего (пиролиза) с образованием пористого остатка и пиролизных газов (от 500 до $1100 \mathrm{~K}$ ); фильтрации пиролизных газов через пористый остаток; вдува пиролизных газов в пограничный слой; уноса массы пористого остатка при достижении наружной поверхностью температуры уноса массы (для различных материалов - различная).

Отсюда видно, что математическое моделирование процессов тепломассопереноса в композиционных материалах представляет собой сложную комплексную проблему, которая в полной мере не решена до сих пор и которой уделяется значительное внимание не только теплофизиками, аэродинамиками, материаловедами, но и математиками. В отношении отдельных частных математических моделей эта проблема решалась в работах [1-5].

До настоящего времени математическое моделирование тепломассопереноса в композиционных материалах осуществлялось для отдельного композиционного материала в силу различных химических составов и различных физико-химических превращений. Попытки разработать универсальные ма-

Екатерина Львовна Кузнецова (к.ф.-м.н., доцент), докторант, каф. вычислительной математики и программирования. 
тематические модели тепломассопереноса, пригодные для любого композиционного материала, не приводили к успеху.

1. Принятые обозначения. В настоящей работе приняты следующие обозначения: $\lambda, c, \rho, T$ - теплопроводность, теплоёмкость, плотность, температура соответственно; $\alpha$ - коэффициент теплоотдачи; $x, t, \delta$ - пространственная и временная переменные, толщина; $\varepsilon$ - степень черноты; П - пористость; $Q$ - теплота фазовых превращений; $u, \bar{M}, R, k, p$ - скорость, молярная масса, газовая постоянная, коксовое число, давление соответственно; $\mu$ - динамическая вязкость.

Индексы у величин применительно к ним означают следующее: ло; к - конец; ** - область пиролиза; г - пиролизный газ; кс - пористый остаток; $s$ - номер слоя $(s=1,2, \ldots, r) ;{ }^{*}$ - наружная граница уноса массы; $w 1$, $w 2$ - наружная и внутренняя свободные границы; $e-$ газ, омывающий свободные поверхности; eff - эффективный.

2. Идентификация закона разложения связующих. В данной работе предложен закон разложения связующих, позволяющий обойти химическую кинетику и пригодный для любого композиционного материала. Существо его заключается в следующем

Для большинства композиционных материалов известны экспериментальные значения температур и плотностей начала $T_{\mathrm{H}}^{* *}, \rho_{\mathrm{H}}$ и окончания $T_{\mathrm{K}}^{* *}, \rho_{\mathrm{K}}$ разложения связующих. Кроме этого, известно также, что изменение плотности связующего композиционного материала описывается экспоненциальным законом с неизвестным предэкспоненциальным множителем и показателем экспоненты, зависящим от температуры и энергии активации. Используя приведенные выше экспериментальные данные, можно определить предэкспоненциальный множитель и показатель экспоненты, которые теперь будут зависеть еще и от времени. Тогда уравнение разложения связующего (аналог закона Аррениуса) можно записать в форме

$$
\begin{gathered}
\frac{d \rho(x, t)}{d t}=\frac{\partial \rho}{\partial t}+\frac{\partial \rho}{\partial x} \frac{d x(t)}{d t}=-\rho A \exp \left(-\frac{B}{T}\right), \\
x_{\mathrm{H}}^{* *}(t) \leqslant x \leqslant x_{\mathrm{K}}^{* *}(t), \quad t>t_{\mathrm{H}}^{* *}
\end{gathered}
$$

где $A$ и $B$ - параметры, подлежащие определению. Для их определения после подстановки в соотношение (1) $T_{\mathrm{H}}^{* *}, \rho_{\mathrm{H}}, T_{\mathrm{K}}^{* *}, \rho_{\mathrm{K}}$ получаем следующую систему уравнений:

$$
\left\{\begin{array}{l}
\frac{\partial \rho_{\mathrm{H}}}{\partial t}+\frac{\partial \rho_{\mathrm{H}}}{\partial x} \dot{x}_{\mathrm{H}}^{* *}=-\rho_{\mathrm{H}} A \exp \left(-B T_{\mathrm{H}}^{* *}\right), \\
\frac{\partial \rho_{\mathrm{K}}}{\partial t}+\frac{\partial \rho_{\mathrm{K}}}{\partial x} \dot{x}_{\mathrm{K}}^{* *}=-\rho_{\mathrm{H}} A \exp \left(-B T_{\mathrm{H}}^{* *}\right),
\end{array}\right.
$$

решая которую, находим:

$$
A=C_{1} \cdot \rho_{\mathrm{H}}\left(\frac{\rho_{\mathrm{H}}}{\rho_{\mathrm{K}}} \frac{C_{1}}{C_{2}}\right)^{-\frac{T_{\mathrm{k}}^{* *}}{T_{\mathrm{K}}^{* *}-T_{\mathrm{H}}^{* *}}}, \quad B=-\ln \left(\frac{\rho_{\mathrm{k}}}{\rho_{\mathrm{H}}} \frac{C_{1}}{C_{2}}\right) \frac{T_{\mathrm{K}}^{* *} T_{\mathrm{H}}^{* *}}{T_{\mathrm{K}}^{* *}-T_{\mathrm{H}}^{* *}},
$$

где

$$
C_{1}=\frac{\rho_{\mathrm{H}}-\rho^{+}}{\tau}+\frac{\left(\rho_{\mathrm{H}}-\rho_{\mathrm{K}}\right) \dot{x}_{\mathrm{H}}^{* *}}{\left(x_{\mathrm{H}}^{* *}\right)^{n}-\left(x_{\mathrm{K}}^{* *}\right)^{n}}, \quad C_{2}=\frac{\left(\rho^{-}-\rho_{\mathrm{H}}\right)\left(\left(x_{\mathrm{H}}^{* *}\right)^{n}-\left(x_{\mathrm{K}}^{* *}\right)^{n+1}\right)}{\left(x_{\mathrm{H}}^{* *}\right)^{n+1}-\left(x_{\mathrm{K}}^{* *}\right)^{n+1}} ;
$$




$$
\begin{aligned}
& \rho^{+}=\rho_{\mathrm{K}}+\frac{\left(\rho_{\mathrm{H}}-\rho_{\mathrm{K}}\right)\left(\left(x_{\mathrm{H}}^{* *}\right)^{n}-\left(x_{\mathrm{K}}^{* *}\right)^{n+1}\right)}{\left(x_{\mathrm{H}}^{* *}\right)^{n+1}-\left(x_{\mathrm{K}}^{* *}\right)^{n+1}}, \\
& \rho^{-}=\rho_{\mathrm{K}}+\frac{\left(\rho_{\mathrm{H}}-\rho_{\mathrm{K}}\right)\left(\left(x_{\mathrm{H}}^{* *}\right)^{n+1}-\left(x_{\mathrm{K}}^{* *}\right)^{n}\right)}{\left(x_{\mathrm{H}}^{* *}\right)^{n}-\left(x_{\mathrm{K}}^{* *}\right)^{n}} .
\end{aligned}
$$

Здесь $\rho^{-}-$значение плотности в $n$-ный момент времени в точке $\left(x_{\mathrm{H}}^{* *}\right)^{n+1}$, т. е. $\rho^{-}=\rho\left(t^{n},\left(x_{\mathrm{K}}^{* *}\right)^{n+1}\right), \rho^{+}$- значение плотности в $(n+1)$-й момент времени в точке $\left(x_{\mathrm{H}}^{* *}\right)^{n}$, т. е. $\rho^{-}=\rho\left(t^{n+1},\left(x_{\mathrm{H}}^{* *}\right)^{n}\right)$.

После определения $A$ и $B$ нелинейное уравнение (1) относительно плотности $\rho$ для зоны разложения связующих $x_{\mathrm{H}}^{* *}(t) \leqslant x \leqslant x_{\mathrm{K}}^{* *}(t)$ решается численно. Из изложенного ясно, что параметры $A$ и $B$ зависят от времени.

3. Обобщенная математическая модель тепломассопереноса в композиционных материалах. Для многослойной области, в которой наружный материал является композиционным, рассматривается следующая задача тепломассопереноса при сложном теплообмене на свободных границах $x_{w 1}, x_{w 2}$ :

- баланс конвективно-кондуктивных и лучистых тепловых потоков на наружной границе $x_{w 1}$, соприкасающейся с высокотемпературным газодинамическим пограничным слоем, с учётом уноса массы:

$$
\begin{gathered}
\alpha_{w 1}\left(T_{e 1}-T_{w 1}\right)-\left.\lambda_{r} \frac{\partial T}{\partial x}\right|_{w 1}-\varepsilon_{w 1} \sigma T_{w 1}^{4}=0, \\
x=x_{w 1}, \quad x_{w 1}=x_{0}+\sum_{s=1}^{r} \delta_{r-s+1}, \quad \lambda=\lambda_{r}, \quad 0<t<t_{\mathrm{H}}^{*} ; \\
\alpha_{w 1}\left(T_{e 1}-T_{w 1}\right)-\left.\lambda_{r} \frac{\partial T}{\partial x}\right|_{w 1}-\varepsilon_{w 1} \sigma T_{w 1}^{4}=\dot{m} Q^{*}\left(T_{e 1}\right), \\
T_{w 1}=T_{w 1}^{*}\left(T_{e 1}\right), \quad \lambda=\left(\lambda_{r}\right)_{\mathrm{eff}}, \quad t_{\mathrm{H}}^{*}<t<t_{\mathrm{K}}^{*}, \quad x=x_{w 1}^{*}(t), \\
x_{w 1}^{*}(t)=x_{0}+\sum_{s=1}^{r} \delta_{r-s+1}-\int_{t_{\mathrm{H}}^{*}}^{t} \dot{x}^{*}(\theta) d \theta ;
\end{gathered}
$$

- уравнение теплопроводности в конструкционных материалах:

$$
\begin{gathered}
c_{s}(T) \rho_{s} \frac{\partial T}{\partial t}=\frac{\partial}{\partial x}\left(\lambda_{s}(T) \frac{\partial T}{\partial t}\right), \\
x_{s-1}<x \leqslant x_{s}, \quad t>0
\end{gathered}
$$

- непрерывность тепловых потоков и температур на границах разрыва теплофизических характеристик конструкционных материалов:

$$
\begin{gathered}
\left.\lambda_{s+1}(T) \frac{\partial T}{\partial x}\right|_{x=x_{s}+0}-\left.\lambda_{s}(T) \frac{\partial T}{\partial x}\right|_{x=x_{s}-0}=0, \\
x=x_{s}, \quad t>0
\end{gathered}
$$

- баланс конвективно-кондуктивных и лучистых тепловых потоков на внутренней границе $x_{w 2}$ :

$$
\begin{gathered}
\alpha_{w 2}\left(T_{e 2}-T_{w 2}\right)+\left.\lambda_{1} \frac{\partial T}{\partial x}\right|_{w 2}-\varepsilon_{w 2} \sigma T_{w 2}^{4}=0, \\
x=x_{w 2}, \quad t>0
\end{gathered}
$$


- уравнение теплопроводности с учётом фильтрации в пористом остатке:

$$
\begin{gathered}
\left(c_{r}\right)_{\mathrm{eff}}(T) \cdot\left(\rho_{r}\right)_{\mathrm{eff}}(x) \frac{\partial T}{\partial t}=\frac{\partial}{\partial x}\left(\left(\lambda_{r}\right)_{\mathrm{eff}}(T) \frac{\partial T}{\partial x}\right)-\Pi\left(c_{\rho} \rho u\right)_{\Gamma} \frac{\partial T}{\partial x}, \\
x_{\mathrm{K}}^{* *}(t)<x<x_{w 1}^{* *}(t), \quad t>t_{\mathrm{H}}^{* *}
\end{gathered}
$$

- температура на границе окончания разложения связующего:

$$
T=T_{\mathrm{K}}^{* *}, \quad x=x_{\mathrm{K}}^{* *}(t), \quad t>t_{\mathrm{K}}^{* *} ;
$$

- уравнение энергии в зоне разложения связующего с учётом физикохимических превращений с тепловым эффектом $Q^{* *}$ и фильтрацией с переменной пористостью:

$$
\begin{aligned}
&\left(c_{r}\right)_{\mathrm{eff}}(x, T) \cdot\left(\rho_{r}\right)_{\mathrm{eff}}(x, T) \frac{\partial T}{\partial t}= \\
&=\frac{\partial}{\partial x}\left(\left(\lambda_{r}\right)_{\mathrm{eff}}(T) \frac{\partial T}{\partial x}\right)-\rho_{r}(x, t) Q^{* *}-\Pi^{* *}(x)\left(c_{\rho} \rho u\right)_{\Gamma} \frac{\partial T}{\partial x}, \\
& x_{\mathrm{H}}^{* *}(t)<x<x_{\mathrm{K}}^{*}(t), \quad t>t_{\mathrm{H}}^{* *} ;
\end{aligned}
$$

- температура на границе начала разложения связующего:

$$
T=T_{\mathrm{H}}^{*}, \quad x=x_{\mathrm{H}}^{* *}(t), \quad t>t_{\mathrm{H}}^{* *} ;
$$

- переменная пористость в зоне разложения связующего:

$$
\Pi^{* *}(x)=\Pi \frac{x-x_{\mathrm{H}}^{* *}}{x_{\mathrm{K}}^{* *}-x_{\mathrm{H}}^{* *}}, \quad x_{\mathrm{H}}^{* *}(t)<x<x_{\mathrm{K}}^{* *}(t), \quad t>t_{\mathrm{H}}^{* *} ;
$$

- идентифицированный закон разложения связующего:

$$
\begin{gathered}
\frac{d \rho_{r}}{d t}=\frac{\partial \rho_{r}}{\partial t}+\frac{\partial \rho_{r}}{\partial x} \frac{d x}{d t}=-\rho_{r} A \cdot \exp \left(-\frac{B}{T}\right), \\
x_{\mathrm{H}}^{* *}(t)<x<x_{\mathrm{K}}^{* *}(t), \quad t>t_{\mathrm{H}}^{* *}
\end{gathered}
$$

- условие Стефана на нестационарно подвижной границе начала разложения связующего:

$$
\begin{gathered}
\left.\left(\lambda_{r}\right)_{\mathrm{eff}}\left(T_{\mathrm{H}}^{* *}\right) \frac{\partial T}{\partial x}\right|_{x=x_{\mathrm{H}}^{* *}(t)+0}-\left.\left(\lambda_{r}\right)_{\mathrm{eff}}\left(T_{\mathrm{H}}^{* *}\right) \frac{\partial T}{\partial x}\right|_{x=x_{\mathrm{H}}^{* *}(t)-0}=\rho_{r} \dot{x}_{\mathrm{H}}^{* *}(t) Q^{* *}, \\
x=x_{\mathrm{H}}^{* *}(t), \quad t>t_{\mathrm{H}}^{* *}
\end{gathered}
$$

- условие Стефана на нестационарно подвижной границе окончания разложения связующего:

$$
\begin{gathered}
\left.\left(\lambda_{r}\right)_{\mathrm{eff}}\left(T_{\mathrm{K}}^{* *}\right) \frac{\partial T}{\partial x}\right|_{x=x_{\mathrm{K}}^{* *}(t)+0}-\left.\left(\lambda_{r}\right)\left(T_{\mathrm{K}}^{* *}\right) \frac{\partial T}{\partial x}\right|_{x=x_{\mathrm{K}}^{* *}(t)-0}= \\
=\left(\rho_{r}\right)_{\mathrm{eff}}\left(x_{\mathrm{K}}^{* *}(t)-0\right) \dot{x}_{\mathrm{K}}^{* *}(t) Q^{* *}, \\
x=x_{\mathrm{K}}^{* *}(t), \quad t>t_{\mathrm{K}}^{* *}
\end{gathered}
$$


- уравнение неразрывности для пиролизных газов:

$$
\frac{\partial\left(p_{\Gamma} u_{\Gamma}\right)}{d x}=0, \quad x_{\mathrm{K}}^{* *}(t)<x<x_{w 1}^{*}(t), \quad t>t_{\mathrm{K}}^{* *}
$$

- уравнение состояния пиролизных газов:

$$
\rho_{\Gamma}(x)=\frac{\rho_{\Gamma}(x) \bar{M}_{\Gamma}}{R_{\Gamma} T(x)}, \quad x_{\mathrm{K}}^{* *}(t)<x<x_{w 1}^{*}(t), \quad t>t_{\mathrm{K}}^{* *}
$$

- закон фильтрации в форме Дарси:

$$
u_{\Gamma}=-\frac{k}{\mu(T)} \frac{d p}{d x}, \quad x_{\mathrm{K}}^{* *}(t)<x<x_{w 1}^{*}(t), \quad t>t_{\mathrm{K}}^{* *}
$$

- давление на наружной свободной границе $x_{w 1}$, давление и плотность торможения пиролизных газов в области разложения связующего:

$$
p\left(x_{w 1}\right)=p_{w 1}, \quad p\left(x_{\mathrm{K}}^{* *}\right)=\frac{\rho_{0 \Gamma} R_{\Gamma} T_{\mathrm{K}}^{* *}}{M_{\Gamma}}, \quad \rho_{0 \Gamma}=\int_{x_{\mathrm{H}}^{* *}}^{x_{\mathrm{K}}^{* *}} \dot{\rho}_{r}(t) d t
$$

- начальные условия для температуры и подвижных границ:

$$
\begin{gathered}
T(x, 0)=T_{\mathrm{H}}(x), \quad x_{0}<x<x_{w 1}, \quad t=0 \\
x_{\mathrm{H}}^{* *}(0)=x_{\mathrm{K}}^{* *}(0)=x_{w 1}^{*}(0)=x_{w 1}
\end{gathered}
$$

- условие необратимости термодинамических процессов:

$$
T_{\mathrm{H}}^{* *}<T_{\mathrm{K}}^{* *}<T_{w 1}^{*}\left(T_{e 1}\right), \quad \rho_{\mathrm{K}}(T)<\rho_{\mathrm{H}}(T) .
$$

Входными данными являются $\alpha_{w 1}(t), T_{e 1}(t), Q^{*}\left(T_{e 1}\right), T^{*}\left(T_{e 1}\right), \varepsilon_{w 1}, \alpha_{w 2}(t)$, $T_{e 2}(t), \varepsilon_{w 2} ; \delta_{s} \lambda_{s}(T), c_{s}(T), \rho_{s} ; \lambda_{\mathrm{Kc}}(T), c_{\mathrm{Kc}}(T), \rho_{\mathrm{Kc}}(T) ; \lambda_{\Gamma}(T), c_{\Gamma}(T), \rho_{\Gamma}(T) ; \rho_{\mathrm{H}}$, $\rho_{\mathrm{K}} ; T_{\mathrm{H}}^{* *}, T_{\mathrm{K}}^{* *}, Q^{* *}, \Pi, \bar{M}_{\Gamma}, k, R_{\Gamma}$.

4. Методология численного решения. Математическая модель (2)-(20) является комплексной, включающей в себя следующие частные задачи:

1) обычного прогрева с граничными условиями различных родов (1-го, 2 -го, 3-го и 4-го рода) при $T(x, t)<T_{\mathrm{H}}^{* *}$;

2) первоначального определения координат подвижных границ;

3) теплопроводности в пористом остатке с учётом фильтрации и подвижных границ - наружной границы уноса массы и границы окончания разложения связующего;

4) генерации пиролизных газов в области разложения связующего и определения плотности и давления торможения $\rho_{O \Gamma}, p\left(x_{\mathrm{K}}^{* *}\right)$;

5) распределения давления газов в пористом остатке, поскольку фильтрация возможна в случае превышения давления в пористом остатке над давлением $p_{w 1}$ окружающей среды;

6) возможного уноса массы с температурой $T^{*}$ и эффектом $Q^{*}$;

7) определения скоростей движения границ $x_{\mathrm{H}}^{* *}(t)$ и $x_{\mathrm{K}}^{* *}(t)$. 
В условиях наличия трех подвижных границ шаг $\tau$ численного интегрирования по времени не может быть принят произвольным, как в случае отсутствия подвижных границ, а должен быть определен из условия ограничения максимальной из всех скоростей движения границ. Для этого можно использовать баланс тепловых потоков (3), поскольку на наружной границе, как правило, тепловые потоки являются максимальными.

Задавая максимальный путь движения границы $x_{w 1}^{*}$ в течение шага по времени, равного, например, шагу $h_{r}$ пространственной сетки, из (3) можно получить верхнюю оценку шага по времени $\tau$ :

$$
\tau \leqslant \frac{h_{r} \cdot \rho_{\mathrm{eff}} \cdot Q^{*}\left(T_{e}\right)}{\alpha_{w 1}\left(T_{e 1}-T_{w 1}^{*}\right)-\left.\lambda_{\mathrm{eff}} \frac{\partial T}{\partial x}\right|_{w 1}-\varepsilon_{w 1} \sigma\left(T_{w 1}^{*}\right)^{4}} .
$$

В силу существенной нелинейности комплексной математической модели (2)-(20) используется разностно-итерационный метод решения. Опишем общий подход.

1. Решается задача теории теплопроводности (2), (4)-(6), (19) в многослойной конструкции с граничными условиями на свободных границах $x_{w 1}, x_{w 2}$ в виде балансов конвективно-кондуктивных и лучистых тепловых потоков. При этом находится распределение температуры $T(x, t)$ в области $x_{0}<x<x_{w 1}$.

2. Если температура $T_{w 1}$ наружной границы $x_{w 1}$ превысит температуру $T_{\mathrm{H}}^{* *}$ начала разложения связующего, то по температуре $T_{\mathrm{H}}^{* *}$ интерполяцией распределения температур определяется начальное положение границы $x_{\mathrm{H}}^{* *}(t)$, которое затем уточняется в итерационном цикле с помощью соотношения (13). Аналогично определяются и начальные значения подвижных границ $x_{\mathrm{K}}^{* *}(t)$ и $x_{w 1}^{*}(t)$ путём интерполяции полученного распределения температур по температурам $T_{\mathrm{K}}^{* *}$ окончания разложения и $T_{w 1}^{*}\left(T_{e 1}\right)$ уноса массы соответственно.

3. Если возникла только граница $x_{\mathrm{H}}^{* *}(t)$, а границы $x_{\mathrm{K}}^{* *}(t)$ и $x_{w 1}^{*}(t)$ не появились, в области $x_{0} \leqslant x \leqslant x_{\mathrm{H}}^{* *}(t)$ решается задача (4)-(6) с граничным условием $(10)$ на границе $x_{\mathrm{H}}^{* *}(t)$. Область $x_{\mathrm{H}}^{* *}(t) \leqslant x<x_{w 1}$ считается областью разложения связующего и в ней решается задача $(2),(9)-(12)$, $(15)-(18)$ с граничным условием (10) на границе $x_{\mathrm{H}}^{* *}(t)$.

4. По найденному распределению температур определяются плотности тепловых потоков на границе $x_{\mathrm{H}}^{* *}(t)$, которые, будучи подставленными в граничное условие Стефана (13) с использованием (10), дают возможность уточнить скорость $\dot{x}_{\mathrm{H}}^{* *}$ движения границы и значение координаты $x_{\mathrm{H}}^{* *}(t)$. Итерационный процесс по уточнению распределения температур и координаты границы $x_{\mathrm{H}}^{* *}(t)$ продолжается до получения заданной точности.

5. При появлении подвижной границы $x_{\mathrm{K}}^{* *}(t)$ окончания разложения связующего в незатронутой разложением области $x_{0} \leqslant x<x_{\mathrm{H}}^{* *}(t)$ решается задача (4)-(6), (10), в нестационарно подвижной области $x_{\mathrm{H}}^{* *}(t) \leqslant$ $\leqslant x<x_{\mathrm{K}}^{* *}(t)$ - задача $(8)-(12),(15)-(18)$, а в области пористого остатка $x_{\mathrm{K}}^{* *}(t)<x<x_{w 1}$ - задача $(2),(7),(8),(15)-(18)$. С помощью полученного распределения температур определяются плотности тепловых потоков на подвижных границах $x_{\mathrm{H}}^{* *}(t)$ и $x_{\mathrm{K}}^{* *}(t)$, подстановка которых в граничные условия Стефана (13) и (14) с учётом (10) и (8) позволяет 
получить линейные скорости $x_{\mathrm{H}}^{* *}(t)$ и $x_{\mathrm{K}}^{* *}(t)$ движения границ области разложения связующего и координат $x_{\mathrm{H}}^{* *}(x), x_{\mathrm{K}}^{* *}(t)$, ограничивающих эту область. Координаты этих границ уточняются в итерационном цикле с заданной точностью.

6. При достижении наружной границей $x_{w 1}$ температуры $T_{w 1}^{*}$ уноса массы возникает подвижная граница $x_{w 1}^{*}(t)$, начальное положение которой определяется интерполяцией распределения температур по температуpe $T_{w 1}^{*}\left(T_{e 1}\right)$. Начиная с этого момента, решаются следующие задачи: в незатронутой области $x_{0} \leqslant x \leqslant x_{\mathrm{H}}^{* *}(t)$ - задача (4)-(6), (10); в области $x_{\mathrm{H}}^{* *}(t) \leqslant x \leqslant x_{\mathrm{K}}^{* *}(t)$ разложения связующего - (8)-(12), (15)-(18) и в области пористого остатка $x_{\mathrm{K}}^{* *}(t) \leqslant x \leqslant x_{w 1}^{*}(t)$-задача (3), (7), (8), (15)-(18), причем на границе $x_{w 1}^{*}(t)$ принимается граничное условие $T_{w 1}=T_{w 1}^{*}\left(T_{e 1}\right)$. Баланс тепловых потоков $(3)$ вместе с условием $T_{w 1}=T_{w 1}^{*}\left(T_{e 1}\right)$ используется для определения массовой $\dot{m}(t)$ и линейной $\dot{x}^{*}(t)$ скоростей движения границы $x_{w 1}^{*}(t)$ после определения температурного поля по граничному условию 1 -го рода $T_{w 1}=T_{w 1}^{*}\left(T_{e 1}\right)$. Итерационный процесс уточняет координаты трёх подвижных границ $x_{\mathrm{H}}^{* *}(t), x_{\mathrm{K}}^{* *}(t), x_{w 1}^{*}(t)$ и распределение температур.

С помощью разработанного программного комплекса был проведён параметрический анализ результатов численного решения задач (2)-(20). При расчётах использовались следующие значения параметров: $T^{*}=1700 \mathrm{~K} ; Q^{*}=$ $=800 \div 1200 \mathrm{\kappa} Д ж / \mathrm{\kappa г} ; Q^{* *}=600 \mathrm{\kappa} Д ж / \mathrm{\kappa г} ; \rho_{\mathrm{H}}=2000 \mathrm{\kappa г} / \mathrm{m}^{3} ; \rho_{\mathrm{K}}=1850 \mathrm{\kappa г} / \mathrm{m}^{3} ;$ $t_{\text {кон }}=40 \mathrm{c} ; \tau=0,2 \mathrm{c} ; \varepsilon_{w 1}=\varepsilon_{w 2}=0,8 ; T_{\text {нач }}=300 \mathrm{~K} ; p_{w 1}=10^{5}$ Па; $r=2$; $\delta_{1}=0,01 \mathrm{~m} ; \delta_{2}=0,01 \mathrm{~m} ; \lambda_{1}=0,0025 \mathrm{KBT} / \mathrm{m} \cdot \mathrm{K} ; \lambda_{2}=0,0004 \mathrm{\kappa BT} / \mathrm{m} \cdot \mathrm{K} ; c_{1}=$ $=1,2 \mathrm{\kappa}$ Дж $/ \mathrm{kr} \cdot \mathrm{K} ; c_{2}=1,6 \mathrm{\kappa}$ Дж $/ \mathrm{kr} \cdot \mathrm{K} ; \rho_{1}=6000 \mathrm{Kг} / \mathrm{m}^{3} ; \rho_{2}=2000 \mathrm{kг} / \mathrm{m}^{3} ; \alpha_{w 1}=$ $=1 \mathrm{KBT} / \mathrm{m}^{2} \cdot \mathrm{K} ; T_{e 1}=3000 \div 4000 \mathrm{~K} ; \alpha_{w 2}=0,2 \mathrm{KBT} / \mathrm{m}^{2} \cdot \mathrm{K} ; T_{e 2}=500 \mathrm{~K} ; \lambda_{\mathrm{Kc}}=$ $=0,002 \mathrm{KBT} / \mathrm{M} \cdot \mathrm{K} ; c_{\mathrm{Kc}}=1,2 \mathrm{~K}$ Дж $/ \mathrm{Kr} \cdot \mathrm{K} ; \rho_{\mathrm{Kc}}=1850 \mathrm{K \Gamma} / \mathrm{m}^{3} ; \lambda_{\Gamma}=0,0001 \mathrm{\kappa BT} / \mathrm{M} \cdot \mathrm{K} ;$ $c_{\Gamma}=1,5 \mathrm{\kappa} Д ж / \mathrm{kг} \cdot \mathrm{K} ; \rho_{\Gamma}=10 \mathrm{kг} / \mathrm{m}^{3} ; \bar{M}=30 \mathrm{\kappa г} /$ кмоль.

На рис. 1 и 2 представлены некоторые результаты этих расчётов в виде зависимостей от времени и температур начала и окончания разложения связующих скоростей движения границ $x^{*}(t) x_{\mathrm{H}}^{* *}(t)$ и $x_{\mathrm{K}}^{* *}(t)$ и погрешностей значения температуры в точке на границе композиционного и конструкционного материалов.

Результаты, представленные на этих рисунках, показали, что если температуры начала и окончания разложения связующего отклоняются от соответствующих экспериментальных значений на 10\%, то максимальный разброс по координатам подвижных границ не превышает $2 \%$, а по температуре в узле $x=x_{w 1}-\delta_{r}$, т.е. на границе между композиционным материалом и подложкой $-4 \%$, причём эти максимальные разбросы соответствуют моментам времени, когда между подвижными границами $x_{\mathrm{H}}^{* *}(t)$ и $x_{\mathrm{K}}^{* *}(t)$ с одной стороны и узлом $x=x_{w 1}-\delta_{r}$ осталось два-три узла фиксированной расчётной сетки.

Таким образом, подтверждается подход к моделированию теплового состояния композиционных материалов, изложенный в данной статье.

Работа выполнена при поддержке ФЦП 1.2.1 ГК П881 от 18.08.2009 ., НОЦ 1.1. ГК 39640-09002 om 16.03.2010 и Грантов Президента Российской Федерации МК-8460.2010.8, HШ-64683.2010.8. 


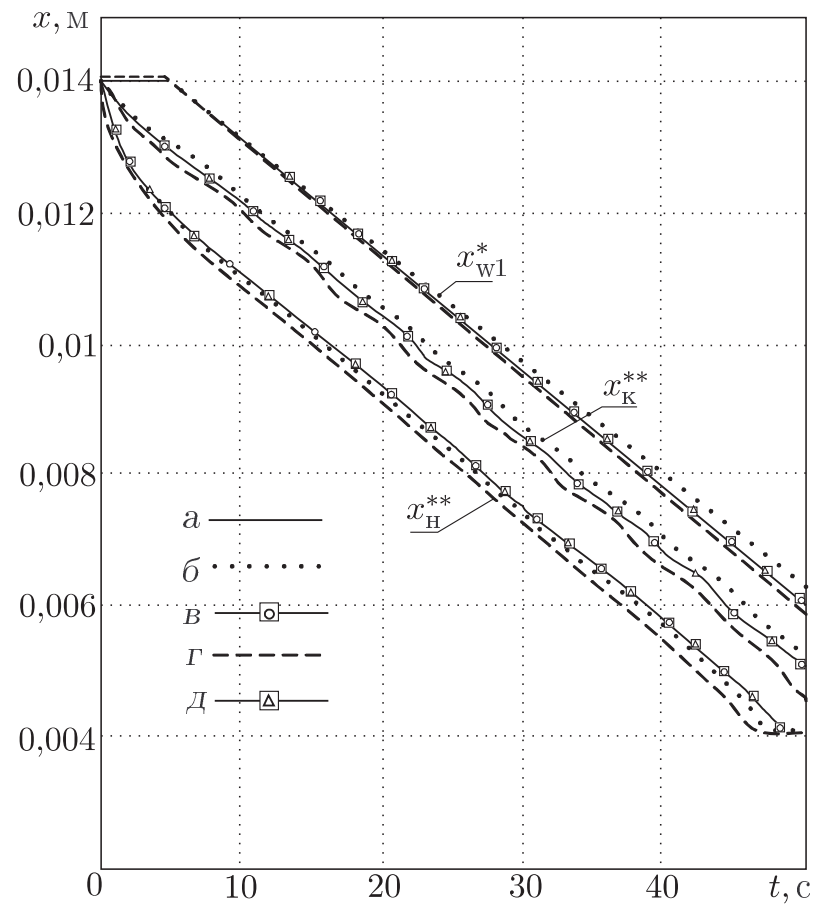

Рис. 1. Изменение по времени координат подвижных границ при изменении температур $T_{\mathrm{H}}^{* *}$ и $T_{\mathrm{K}}^{* *}:$ a) $T_{\mathrm{H}}^{* *}=500 \mathrm{~K}, T_{\mathrm{K}}^{* *}=1100 \mathrm{~K}$; б) $T_{\mathrm{H}}^{* *}=525 \mathrm{~K}, T_{\mathrm{K}}^{* *}=1100 \mathrm{~K} ;$ в) $T_{\mathrm{H}}^{* *}=550 \mathrm{~K}$ $T_{\mathrm{K}}^{* *}=1100 \mathrm{~K} ;$; $) T_{\mathrm{H}}^{* * *}=500 \mathrm{~K}, T_{\mathrm{K}}^{* *}=1045 \mathrm{~K} ;$ д) $T_{\mathrm{H}}^{* *}=525 \mathrm{~K}, T_{\mathrm{K}}^{* *}=1145 \mathrm{~K}$

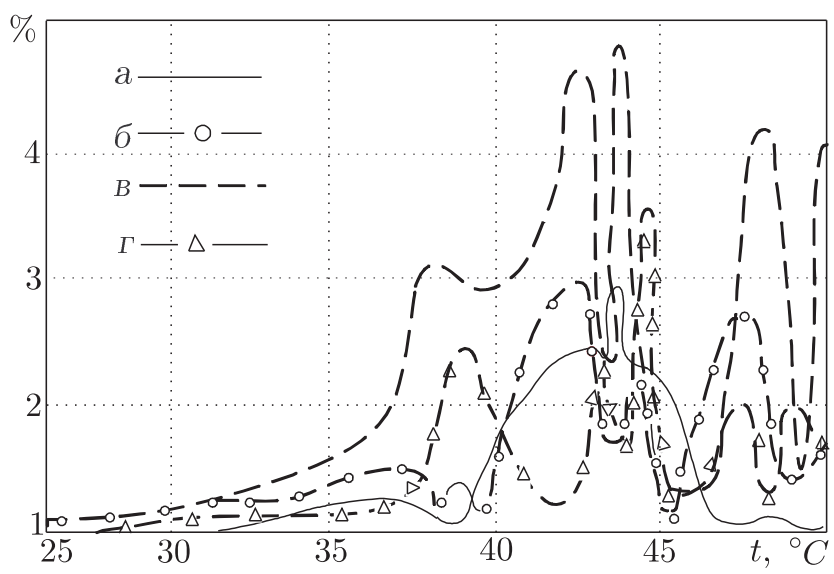

Рис. 2. Изменение по времени относительной температуры в точке $x=x_{w 1}-\delta_{r}$ относительно значений $T_{\mathrm{H}}^{* *}=500 \mathrm{~K}, T_{\mathrm{K}}^{* *}=1100 \mathrm{~K}$ при следующих изменениях $T_{\mathrm{H}}^{* *}$ и $T_{\mathrm{K}}^{* *}:$ а $T_{\mathrm{H}}^{* *}=525 \mathrm{~K}, T_{\mathrm{K}}^{* *}=1100 \mathrm{~K} ; \sigma-T_{\mathrm{H}}^{* *}=500 \mathrm{~K}, T_{\mathrm{K}}^{* *}=1100 \mathrm{~K} ; B-T_{\mathrm{H}}^{* *}=500 \mathrm{~K}, T_{\mathrm{K}}^{* *}=1045 \mathrm{~K}$; $\Gamma-T_{\mathrm{H}}^{* *}=525 \mathrm{~K}, T_{\mathrm{K}}^{* *}=1145 \mathrm{~K}$ 


\section{БИБЛИОГРАФИЧЕСКИЙ СПИСОК}

1. Кудинов В.А., Аверин Б.В., Стефанюк Е.В., Назаренко С.А. Анализ нелинейной теплопроводности на основе определения фронта температурного возмущения // TBT, 2006. - T. 44, № 5. - C. 577-585.

2. Кудинов B. А., Стефанюк E. В. Задачи теплопроводности на основе определения фронта температурного возмущения // Известия АН. Энергетика, 2008. - № 4. - С. 122-138.

3. Полежаев Ю. В., Юревич Ф. Б. Тепловая защита. - М.: Энергия, 1976. - 392 с.

4. Формалев В. Ф. Тепломассоперенос в анизотропных телах. Обзор // TBT, 2001. - Т. 39, № 5. - C. 810 .

5. Формалев В.Ф., Колесник С.А., Миканев С.В. Моделирование теплового состояния композиционных материалов // ТВT, 2003. - Т. 41, №6. - С. 935-341.

Поступила в редакцию $23 / \mathrm{XI} / 2009$;

в окончательном варианте - 30/VIII/2010.

MSC: 80A17, 80M25

\section{A COMPOSITE HEAT STATE MODELLING BASED ON UNIVERSAL DECOMPOSITION COMPOSITES RULE}

\section{K. L. Kuznetcova}

Moscow Aviation Institute (State University of Aerospace Technologies),

4, Volokolamskoe Shosse, Moscow, 125993, Russia.

E-mail: lareyna@mail.ru

The mathematical model and numerical method to solving the problems of heat state of majority composites in conditions of an appreciably unsteady high-thermal stressing are offered. Its base on the identification of the decomposition composites rule. This rule has been obtained and let to exclude chemical kinetics in a region of the decomposition in problems about heat state of the composition. Analysis of the numerical results proves this approach to modeling of heat state composites to be a proper one.

Key words: composite, fine-fibred filler, resin binder, heat and mass transfer, mathematical modeling, numerical methods.

Original article submitted 23/XI/2009; revision submitted $30 / \mathrm{VIII} / 2010$.

Ekaterina L. Kuznetcova (Ph. D. (Phys. \& Math.)), Doctoral Candidate, Dept. of Computational Mathematics \& Programming. 